\title{
Search for dark matter in the hidden-photon sector with a large spherical mirror
}

\section{Darko Veberič $^{* a}$, Kai Daumiller ${ }^{a}$, Babette Döbrich ${ }^{b}$, Ralph Engel ${ }^{a}$, Joerg Jaeckel ${ }^{c}$ Marek Kowalski ${ }^{d e}$, Axel Lindner $^{d}$, Hermann-Josef Mathes ${ }^{a}$, Javier Redondo ${ }^{f}$, Markus Roth ${ }^{a}$, Christoph Schäfer ${ }^{a}$, Ralf Ulrich $^{a}$ [The FUNK Experiment]}

${ }^{a}$ Institute for Nuclear Physics, Karlsruhe Institute of Technology (KIT), Germany

${ }^{b}$ Physics Department, CERN, Geneva, Switzerland

${ }^{c}$ Institute for Theoretical Physics, Heidelberg University, Germany

${ }^{d}$ Deutsches Elektronen Synchrotron DESY, Hamburg, Germany

${ }^{e}$ Department of Physics, Humboldt University, Berlin, Germany

${ }^{f}$ Department of Theoretical Physics, University of Zaragoza, Spain

*E-mail: darko.veberic@kit.edu

If dark matter consists of hidden-sector photons which kinetically mix with regular photons, a tiny oscillating electric-field component is present wherever we have dark matter. In the surface of conducting materials this induces a small probability to emit single photons almost perpendicular to the surface, with the corresponding photon frequency matching the mass of the hidden photons. We report on a construction of an experimental setup with a large $\sim 14 \mathrm{~m}^{2}$ spherical metallic mirror that will allow for searches of hidden-photon dark matter in the $\mathrm{eV}$ and sub-eV range by application of different electromagnetic radiation detectors. We discuss sensitivity and accessible regions in the dark matter parameter space.

The 34th International Cosmic Ray Conference

30 July - 6 August, 2015

The Hague, The Netherlands

\footnotetext{
* Speaker.
} 



Figure 1: Left: A 6×6-segmented spherical mirror [8], the same as used for the fluorescence-detector telescopes of the Pierre Auger Observatory, where each segment is $65 \mathrm{~cm}$ high with width gradually decreasing from $65 \mathrm{~cm}$ to $55 \mathrm{~cm}$. Right: View of the support structure for the segments at the back of the mirror. The center of the mirror sphere is also marked with a point.

\section{Introduction}

There is a number of convincing astrophysical and cosmological evidences that a large fraction of the energy density in the universe must be composed of invisible non-baryonic matter or dark matter (DM) [1]. The most explored options for explaining DM are extensions of the Standard Model (SM) predicting axions and weakly-interacting massive particles. In recent years attention has been turned also to alternatives or weakly-interacting slim particles (WISP), as e.g. axion-like particles or hidden photons (HP). WISP could be nonthermally produced in the early universe and survive as cold DM until today.

Here we consider HP, which are additional light U(1) gauge bosons that kinetically mix with the (vis-à-vis uncharged) SM particles [2]. The effective Lagrangian can be written as

$$
\mathscr{L}=-\frac{1}{4}\left(F_{\mu v} F^{\mu v}+X_{\mu v} X^{\mu v}\right)+J^{\mu} A_{\mu}+\frac{m^{2}}{2} X_{\mu} X^{\mu}-\frac{\chi}{2} F_{\mu v} X^{\mu v},
$$

where $F_{\mu \nu}=\partial_{\mu} A_{v}-\partial_{v} A_{\mu}$ is the field strength tensor of the photon and $X_{\mu \nu}$ that of the corresponding HP field, and $J^{\mu}$ the electric current. Large regions of the HP mass $m$ and mixing parameter $\chi$ space are compatible with the observed DM signatures [3, 4] and are already searched for with many experimental methods: haloscopes, helioscopes, and light-shining-through-a-wall methods [3]. Our experiment, Finding U(1)s of a Novel Kind (FUNK), is dedicated to observe possible candidates for HP with the dish-antenna method [5, 6], where the faint electromagnetic waves, emitted almost perpendicularly to conducting surfaces, are focused by using a spherically shaped mirror. The HP induced light emerging from any part of the mirror is thus gathered in the center of the sphere where various detectors can be suitably placed. The DM nature of the signal can be verified by observation of the expected small daily and seasonal movements of the spot [7]. 



Figure 2: Left: Frosted glass screen (with a SMD LED on the front face), linear stage with stepper motor, and a Raspberry Pi micro-computer as controller. Right-top: Schematical description of the sphere-center reference point with two crossing laser beams (red along the mirror axis, green laterally). Mirror segments were adjusted until focused and converging into this point. Right-bottom: Photo of the frosted glass and the crossing laser beams as seen in darkness.

\section{Mirror}

For the purposes of the FUNK experiment we reused spherical mirror components that were originally produced for the Schmidt telescopes of the fluorescence detector of the Pierre Auger Observatory [8]. At the KIT Campus Nord we assembled them inside of a windowless air-conditioned experimental hall of $\sim 20 \mathrm{~m}$ diameter with $2 \mathrm{~m}$ thick concrete walls. The whole mirror is composed of three different segment shapes that are arranged into a $6 \times 6$ matrix (see Fig. 1). The height of the segments is exactly $65 \mathrm{~cm}$ but moving away from the mirror equator their width gradually decreases from $65 \mathrm{~cm}$ to $55 \mathrm{~cm}$. The mirror segments were produced by milling a cast aluminium backing to approximate spherical shape and gluing it at high temperatures with a thinner AlMgSi sheet [9]. The front reflective surface was then precisely milled with diamond tools and protected with electrochemical anodization, resulting in a final reflectivity of $88 \pm 1 \%$ in visible and UV range. The segments are attached to the mirror support structure via single-point ball joint. The extreme four corners of the mirror form a $3.7 \times 3.1 \mathrm{~m}$ rectangle, nevertheless, due to the spherical geometry with radius $3.4 \mathrm{~m}$, the total area of the mirror front surface is $14.56 \mathrm{~m}^{2}$.

The initial assembly of the mirror was performed with help of an existing laser tool according to the specifications used for the telescopes of the Auger Observatory. Unfortunately, the prototype components from the construction were mirror segments which mainly did not pass strict quality requirements for their radius of curvature or other relevant parameters. To quantify the optical 

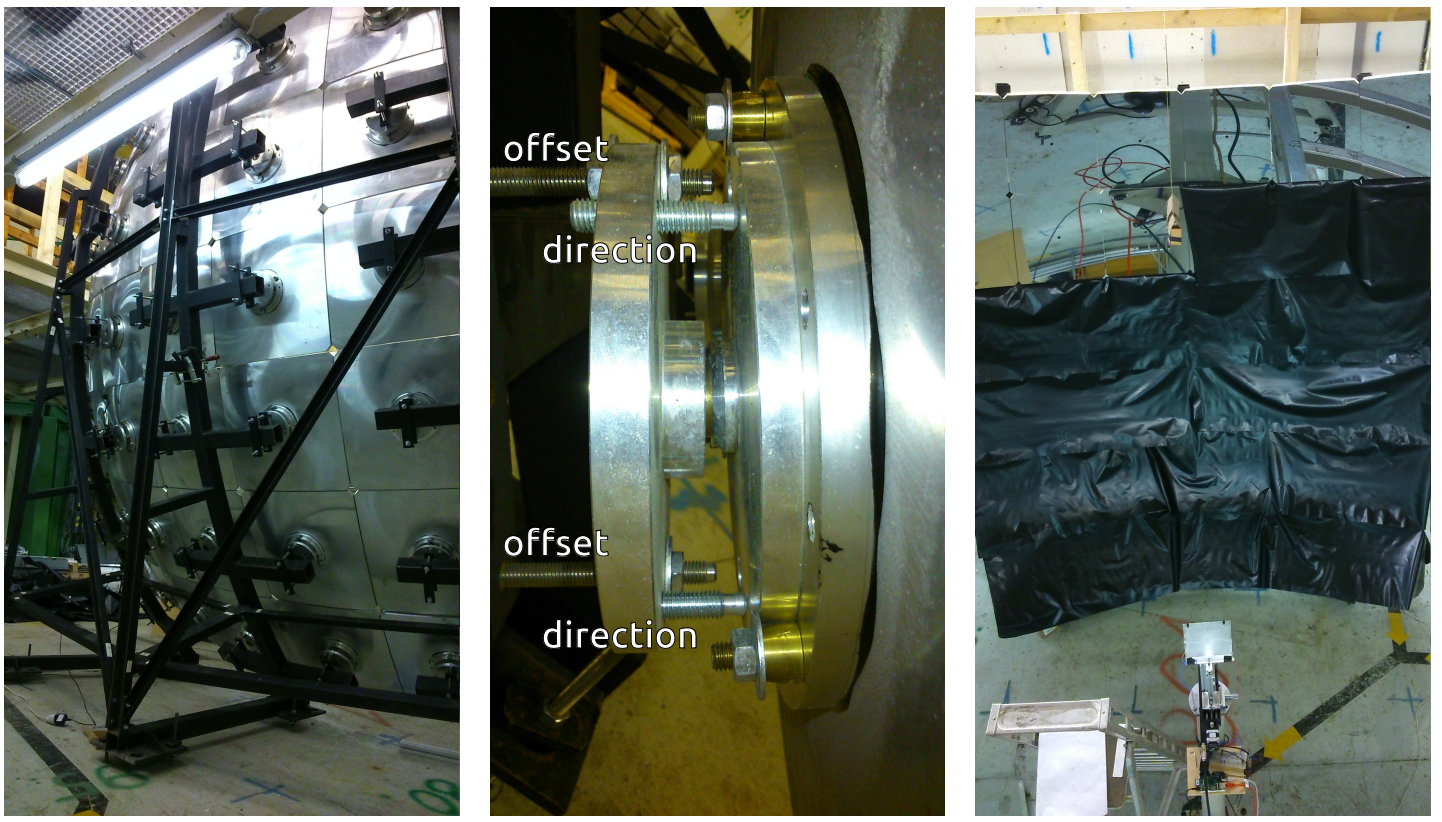

Figure 3: Left: Back side of the mirrors exposing the mounts of the segments. Middle: Screws enabling longitudinal offset movement and fine setting of the pointing direction via three-point tilting of the ball joint. Right: Already aligned segments are covered with non-reflecting foil to keep only the misaligned beams in the reference point. At the bottom of the photo the frosted-glass screen can be seen placed on a linear stage.
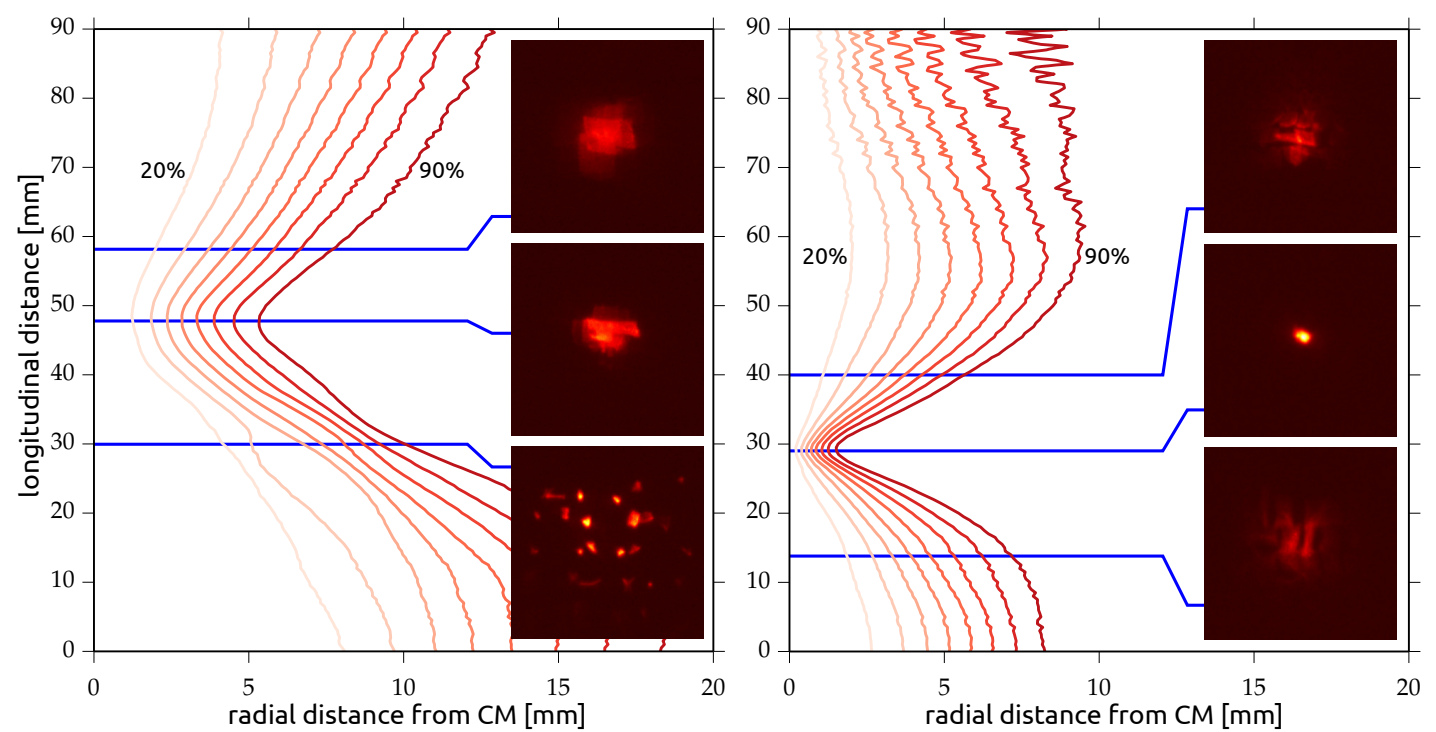

Figure 4: Left: Contours of radial distance from the image center of mass (CM) containing certain amount of total light (in steps of $10 \%$ ) for the initial assembly of the mirror. The three insets show spot images in various positions marked by blue lines (middle for smallest spread). Note the focusing of individual beams from segments but without their convergence (bottom inset). Right: The same as on the left but after the realignment.

quality of the mirror we built a movable imaging platform at the center of the mirror sphere (see Fig. 2-left). It consists of a frosted-glass screen with a yellow-green SMD LED as a light source. 

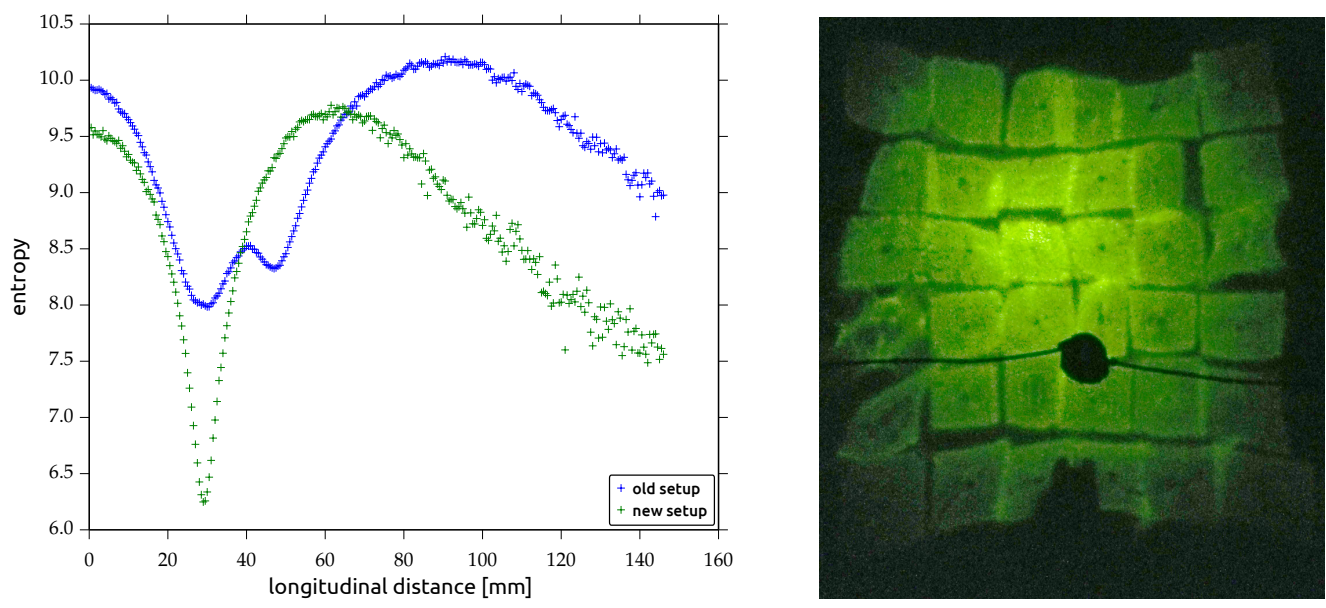

Figure 5: Left: Entropy of the image at different longitudinal positions of the screen (blue points for the initial mirror assembly and green points after the readjustment of individual segments). Right: An image obtained when the LED is driven off-center towards the mirror. Reflected beams are not converged yet so that they form a matrix where each of the squares corresponds to individual mirror segments, revealing their aberrations. The central black dot is a shadow of the LED with two horizontal electricity cables.

Note that in the case of an ideal spherical mirror, light from a point source placed exactly in the center of the sphere will be reflected and focused back onto the source itself. With a slight lateral offset of the source the resulting image can be observed in the screen next to it, with the offsetrelated distortions being very small. This reflected light pattern was then observed with a CCD camera. Placing the screen on a movable linear stage enabled us to obtain cross-section images of the converging light beams from the mirror segments in various longitudinal positions along the optical axis of the mirror. In Fig. 4 such a scan through the central region is presented with contour lines for distances (from the image center of mass) containing certain fractions of the total light (in 10\% steps). As seen in Fig. 4-left, the initial assembly of the mirror produced in a minimal region (middle inset) a very large focal spot size with radius $\sim 6 \mathrm{~mm}$ (for $90 \%$ of total light). We also observed that most of the individual segments had good focus points but at distances different than the nominal radius (see lower inset), indicating that by realigning individual segments we can achieve better convergence and smaller final spot size. This position was chosen as a new dedicated mirror center and permanently marked for future reference with two crossing laser beams (see Fig. 3-right) which will later-on also help to accurately position the measuring equipment.

In a dedicated readjustment campaign we aligned all mirror segments, either by offsetting them longitudinally or changing their pointing direction (see Fig. 3) until the sharpest possible spot has been produced by the corresponding segment. In this way we compensated for the non-nominal values of the curvature while other aberrations, nicely seen in an off-center image in Fig. 5-right, remained. Nevertheless, out of 36 segments, we identified only 5 that had the individual spot sizes considerably larger than the LED source size, mainly due to having two different radii of curvature in the two perpendicular lateral directions, in Fig. 5-right identified as having more rectangular instead of square shapes. The $90 \%$ spot radius now decreased to only $\sim 2 \mathrm{~mm}$ which enabled us to use a small CCD as a detector. Note that the seasonal oscillation of the HP spot due to the relative movement of the mirror in the DM frame is of the same order [6]. A way to quantify the 

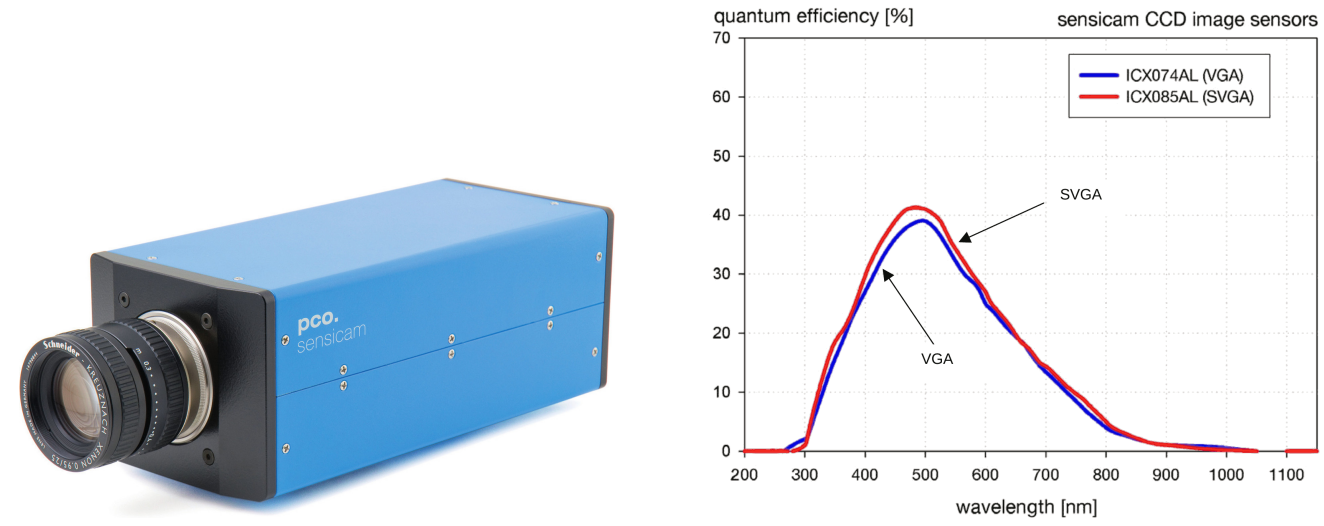

Figure 6: Left: PCO Sensicam camera used for the preliminary measurement. Right: Quantum efficiency of the camera CCD (see blue VGA curve) from [10].
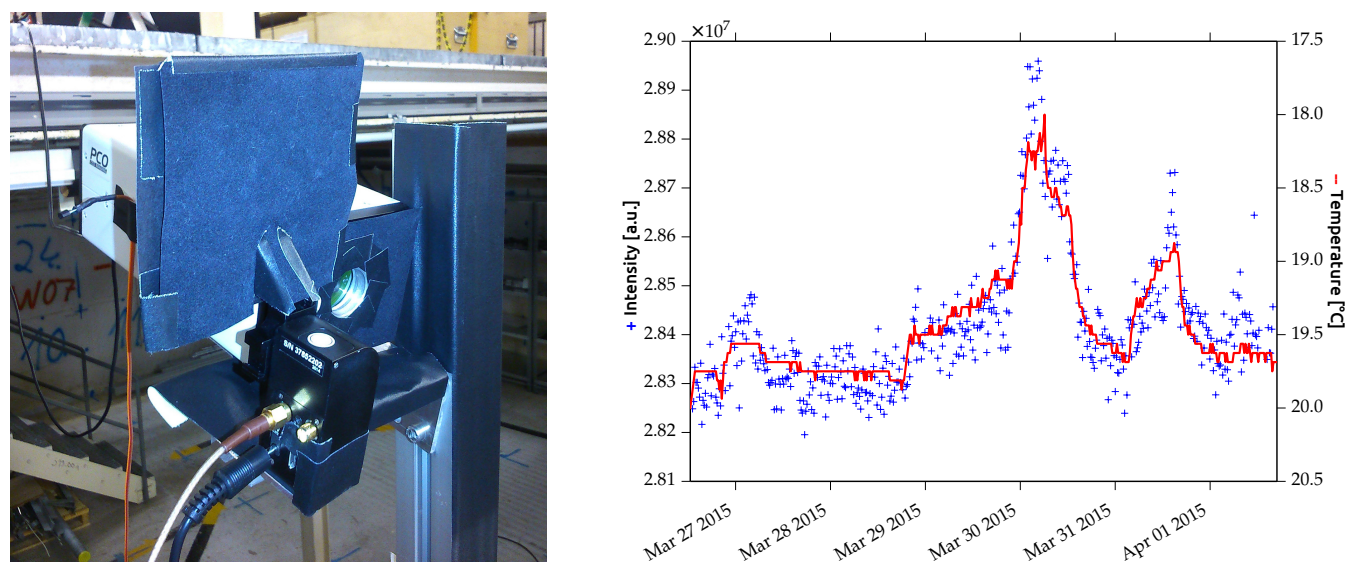

Figure 7: Left: The mounted camera with removed lens and its CCD placed in the reference point. The Thorlabs Motorized Filter Flipper is used to drive a shutter. Right: The evolution of intensity (blue) with time in a longer example run. Long term deviations are due to temperature (red) dependence of the CCD intensity.

sharpness of the image with a single observable is using entropy, defined as $S=-\sum_{i} p_{i} \ln p_{i}$ where $p_{i}$ is the (normalized) intensity in image pixel $i$. From the entropy an effective number of pixels or area containing relevant signal can be defined as $N_{\text {eff }}=\exp (S)$. In Fig. 5-left, entropy of the longitudinal scan images are compared. While the initial mirror setup was exhibiting two entropy minima, a deeper one when individual segments were focused and a shallower one for when the individual beams crossed $20 \mathrm{~mm}$ away, the entropy after the readjustment shows only one deep minimum with at least 6 times smaller effective spot area.

\section{Preliminary results}

After the final preparation of the mirror we performed preliminary measurements with the CCD camera that was used to obtain the images for Fig. 4 and 5 scans. The camera is a PCO sensicam with a half-inch diameter, VGA resolution $(640 \times 480)$ CCD sensor, internally Peltiercooled to $-15^{\circ} \mathrm{C}$. The quantum efficiency is given in Fig. 6. The collected charge to pixel count is 

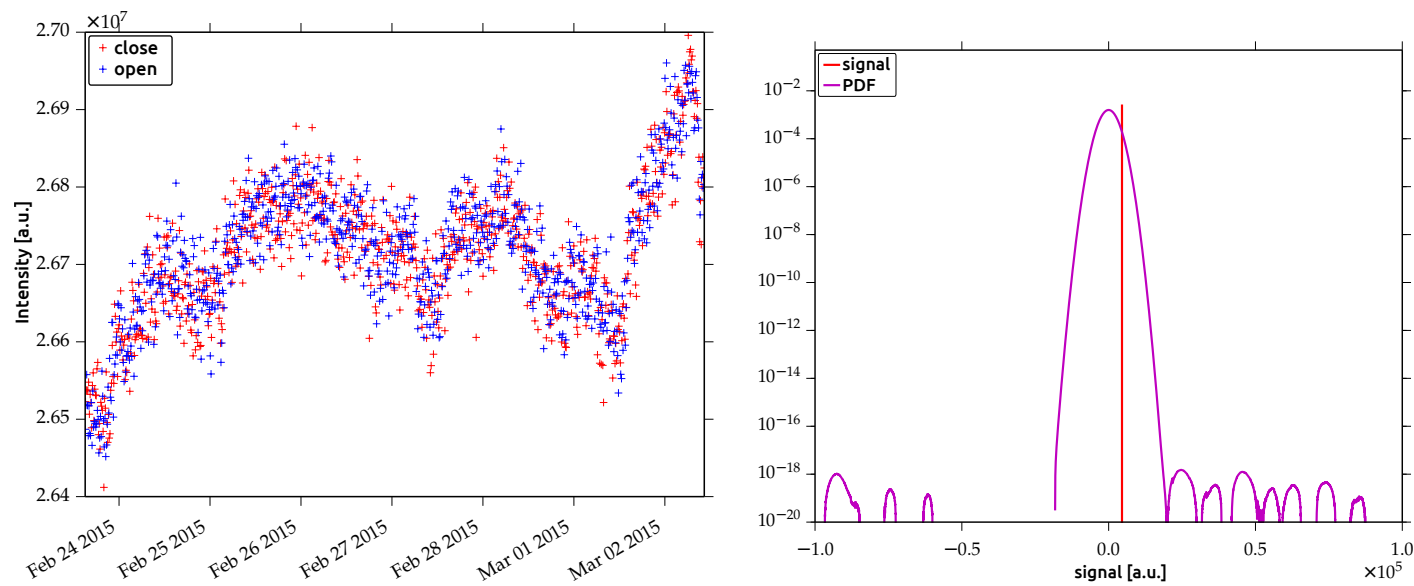

Figure 8: Example of a 7 day measurement run with $1000 \mathrm{~s}$ exposures. Left: Total image intensities for open (blue) and closed (red) shutter. Right: Expected distribution of intensity differences (PDF) is shown in violet (side lobes are artifacts of the FFT convolution) and the total observed difference of the run with red vertical line).

specified [10] at 7.5 $e^{-}$/count, pixel readout noise is $\sim 14 e^{-}$, and dark current per pixel is $<0.1 e^{-} / \mathrm{s}$. We removed the lens, placed the CCD into the reference point, and added a motorized filter flipper, see Fig. 7-left, to drive a large shutter. Since the spot size is not much smaller than the whole $\mathrm{CCD}$, the relevant quantity for this sensor is the total intensity (integral over all pixels of the CCD). In Fig. 7-right an example of a long-term intensity measurement with a closed shutter is shown, together with the temperature of the camera enclosure (red), revealing a temperature correlation on the order of $-1 \% / \mathrm{K}$. In Fig. 8-left an example of image intensities (in one of many long-term measurement runs we performed) is shown for open and closed shutter. The signal is defined as the difference of these two measurements. In Fig. 8-right a distribution of possible signals is shown based on noise properties of individual images. The actual signal is shown with a red vertical bar, being perfectly compatible with the estimated noise.

No signal from HP is thus observed, allowing us to set in this test example preliminary upper limits [7] on the mixing parameter $\chi$ in the sensitivity range of the CCD, see Fig. 9.

\section{Conclusions and future plans}

As a first step of the FUNK experiment we assembled the segments of a prototype mirror of the Pierre Auger Observatory to obtain a large $\sim 14 \mathrm{~m}^{2}$ spherical mirror, suitable for dark matter searches in the hidden photon sector. With manual readjustments we managed to reduce the radius of the spot size below $\sim 2 \mathrm{~mm}$ which enables us to use small and highly sensitive detectors of electromagnetic radiation.

While the initial tests were made with a simple CCD camera we are preparing high-sensitivity measurements with a low dark-current CCD and a low noise PMT. Furthermore, in the near future we want to extend the search for possible $\mathrm{HP}$ mass into the $\mathrm{MHz}$ [11], GHz [12], and $\mathrm{THz}$ range.

We gratefully acknowledge partial funding from the the Helmholtz Alliance for Astroparticle physics (HAP), funded by the Initiative and Networking Fund of the Helmholtz Association. 


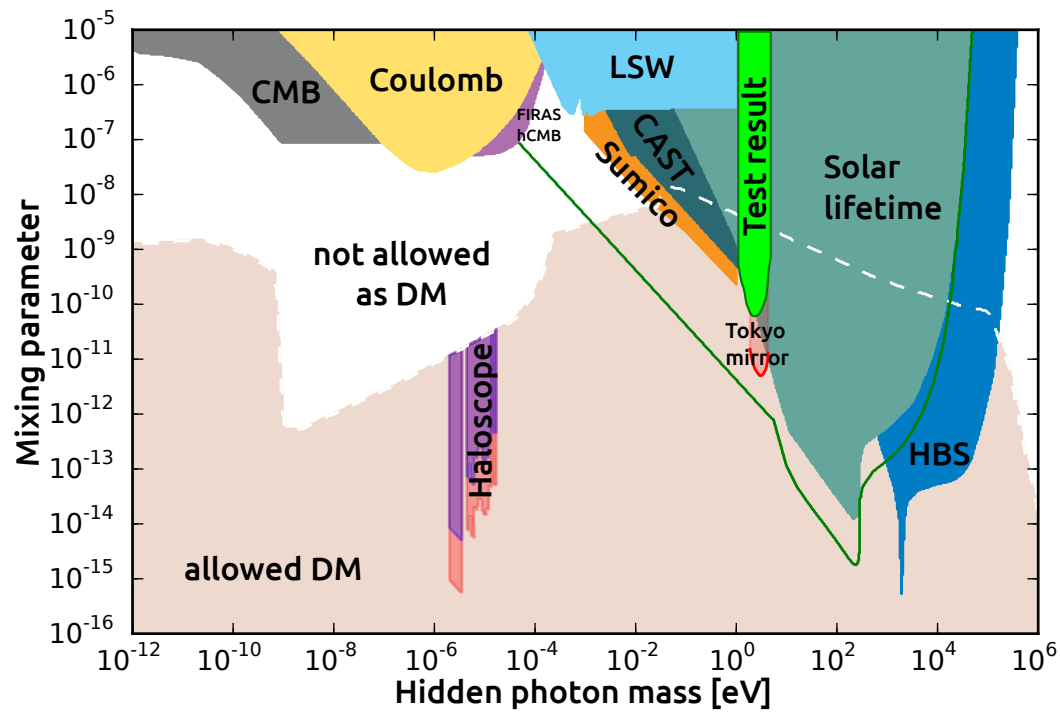

Figure 9: Exclusion plot in the mixing parameter vs. hidden photon mass parameter space. Meaning of some labels: measurements on cosmic microwave background (CMB), light-shining-through-wall experiments (LSW), horizontal-branch stars (HBS). Our preliminary result with the CCD camera is shown in light green (Test result). Plot adapted from [13] (Tokyo mirror), for summary and labels see [14].

\section{References}

[1] G.B. Gelmini, TASI 2014 lectures: The hunt for dark matter, [arXiv: 1502.01320].

[2] K. Baker et al., The quest for axions and other new light particles, Ann. Phys. 525 (2013) A93-A99.

[3] P. Arias, D. Cadamuro, M. Goodsell, J. Jaeckel, J. Redondo, and A. Ringwald, WISPy cold dark matter, JCAP 06 (2012) 013

[4] P.W. Graham, J. Mardon, and S. Rajendran, Vector Dark Matter from Inflationary Fluctuations, [arXiv: 1504.02102].

[5] D. Horns, J. Jaeckel, A. Lindner, A. Lobanov, J. Redondo, and A. Ringwald, Searching for WISPy Cold Dark Matter with a Dish Antenna, JCAP 04 (2013) 016.

[6] J. Jaeckel and J. Redondo, An antenna for directional detection of WISPy dark matter, JCAP 111 (2013) 016.

[7] B. Döbrich, K. Daumiller, R. Engel, M. Kowalski, A. Lindner, J. Redondo, and M.Roth, Hidden Photon Dark Matter Search with a Large Metallic Mirror, Proc. 10th Patras Workshop on Axions, WIMPs and WISPs, CERN, 29 June - 4 July 2014, [arXiv: 1410.0200].

[8] J. Abraham et al. [Pierre Auger Collaboration], The Fluorescence Detector of the Pierre Auger Observatory, Nucl. Instrum. Meth. A $\mathbf{6 2 0}$ (2010) 227-251.

[9] J. Abraham et al. [Pierre Auger Collaboration], Properties and performance of the prototype instrument for the Pierre Auger Observatory, Nucl. Instrum. Meth. A 523 (2004) 50-95.

[10] Sensicam, Sensicam QE, Sensicam EM - Operating Instructions, PCO Imaging (2004); http://www.pco.de.

[11] A. Aab et al. [Pierre Auger Collaboration], Probing the radio emission from air showers with polarization measurements, Phys. Rev. D 89 (2014) 052002.

[12] R. Šmída et al. [CROME experiment], First Experimental Characterization of Microwave Emission from Cosmic Ray Air Showers, Phys. Rev. Lett. 113 (2014) 221101.

[13] J. Suzuki, T. Horie, Y. Inoue, and M. Minowa, Experimental Search for Hidden Photon CDM in the eV mass range with a Dish Antenna, [arXiv: 1504.00118].

[14] M. Goodsell, J. Jaeckel, J. Redondo, and A. Ringwald, Naturally light hidden photons in LARGE volume string compactifications, JHEP 11 (2009) 027. 